\title{
Where We're Headed: A New Wave of Scholarship on Educating Medical Professionalism
}

\author{
Paul Haidet, $\mathrm{MD}, \mathrm{MPH}^{\top}$ \\ ${ }^{1}$ The Houston Center for Quality of Care and Utilization Studies at the DeBakey Veterans Affairs Medical Center and Baylor College \\ of Medicine, Houston, TX, USA.
}

\author{
KEY WORDS: education, medical; physician's role; education, medical, \\ undergraduate; education, medical, graduate; social environment; \\ organizational culture; schools, medical; internship and residency; \\ professional practice; ethics, professional; professional-patient \\ relations; learning; teaching; psychology, educational. \\ J Gen Intern Med 23(7):1118-9 \\ DOI: $10.1007 / \mathrm{s} 11606-008-0670-5$ \\ (c) Society of General Internal Medicine 2008
}

\begin{abstract}
A $\mathrm{h}$ yes, another editorial on professionalism. This topic has certainly been one of accelerating interest. For example, a PubMed search on the title word "professionalism" finds 1331 articles as outlined in Table 1; approximately 100 articles per year have appeared since the 2002 publication of the physician charter. ${ }^{1}$ While much of the past literature has focused on the definition of professionalism, increasingly, the field is moving in the needed direction of discovering and building processes that foster professional attitudes and behaviors among physicians and physicians in training. In this education issue of The Journal of General Internal Medicine, at least six articles focus on the subject. ${ }^{2-7}$ While all six add to the literature in their own right, taken collectively, they contain several powerful messages that provide a beacon for future work in this field:
\end{abstract}

\section{PROFESSIONALISM IS NOT BOUNDED BY THE ENDS OF THE WORKDAY}

When addressing professional attributes such as compassion, altruism, trustworthiness, duty, and others, many articles tend to focus mainly on what happens in the life-space of medicine. An implicit assumption in such articles is that physicians who behave professionally do so because that is an inherent part of their makeup, and such attributes will seamlessly cross into their lives outside of medicine. However, when we look into that outside world, this may not be the case. Thompson and colleagues' findings of professionally challenging content on the Facebook pages of medical trainees suggests that at least some students may be living different lives inside and outside of the medical school. ${ }^{2}$ On a more positive note, Wear and Zarconi found important foundational influences on professional attitudes and behaviors in the life-

The opinions expressed herein reflect those of the author and do not necessarily represent the views of the U.S. Department of Veterans Affairs or Baylor College of Medicine. worlds of students prior to coming to medical school. ${ }^{3}$ These studies raise some important questions. Is professionalism like a white coat that physicians put on at the beginning of the day and take off when they go home, or should it be a set of principles for physicians to use to guide their lives? How can we educators help students and trainees to find an authentic voice, ${ }^{8}$ based at least in part on the profession's ideals, that works in both medical and nonmedical life-worlds? Are physicians who can be their authentic selves during the workday (as opposed to playing a role that denies part of their personhood) less likely to experience burnout? ${ }^{9}$ Are Medical College Admissions Test scores and grade point averages the best proxy to assess for foundational influences that are harmonious with the values of our profession? If not, are other methods (quantitative, qualitative or otherwise) to assess these influences possible? A rich set of opportunities awaits the educators who: a) seek to understand the complex interplay between influences inside and outside of the medical world, and b) use the lessons learned to influence the processes (e.g., admissions, curriculum, and others) of medical education.

\section{SELF-REFLECTION IS AN IMPORTANT PATHWAY TO PROFESSIONALISM}

Several of the articles in this issue describe curricula to foster professionalism. ${ }^{3-5}$ All are based on reflection. Both HillSakurai and colleagues' longitudinal intersession-based third-year curriculum, and Fisher and colleagues' description of multiple institutions' internal medicine clerkship activities, contain a central component that includes written reflection on students' experiences and the meanings of those experiences for students' emerging professional identities. Similarly, Wear and Zarconi's data were based on a 'capstone' writing assignment intended to collect data about the curriculum, and the examples also show evidence of possible new insights by the students who wrote them. It is not a coincidence that many curricula about professionalism include reflective activities. It is also not a coincidence that Inui's A Flag In the Wind, ${ }^{10}$ perhaps the most comprehensive and definitive statement on professionalism education to date, begins each one of its major sections with a personal reflection from Dr Inui's own career. Self-reflection is a pathway to growth, and professionalism education is all about growth. It is fitting, then, that the body of scholarship on professionalism also begin to include scholarship that advances the practice and creation of selfreflection. Much work remains to be done. What cognitive and emotional processes occur when a student self-reflects? How do such processes present opportunities for educators to 
Table 1. English Language Articles Published With the Word 'Professionalism' in the Title'

\begin{tabular}{lc}
\hline \hline Time Period & Number of Articles \\
\hline $2000-2008$ & 701 \\
$1990-2000$ & 270 \\
$1980-1990$ & 190 \\
$1970-1980$ & 135 \\
$1965-1970$ & 35 \\
\hline
\end{tabular}

* Based on a PubMed search using the term "Professionalism," Limits "English Language," and Default Tag "Title", performed 5/1/08. Time periods run from June 1 of the earlier year to May 31 of the later year

create reflective moments? Can we assess the quality of reflection (an inherently inward process) by observing outward behaviors or artifacts, such as writing assignments or portfolios? Is it possible for a teacher to turn everyday, mundane events (such as small acts of humanism) into powerful sentinel events? Can learners be diagnosed in terms of their "selfreflectiveness," and do different levels of reflectiveness require different teaching strategies? As with the inside/outside issue above, great opportunities exist to better understand the processes of self-reflection and their place in enhancing professionalism in medical education.

\section{PROFESSIONALISM IS AS MUCH A PRODUCT OF THE SYSTEM AS IT IS OF THE INDIVIDUAL}

This last statement captures the essence of the hidden curriculum. It is not surprising that Ginsburg and colleagues ${ }^{6}$ found themes like: "do what you're told;" these themes are congruent with underlying messages, such as "hierarchy is necessary," that exist in the organizational cultures of medical schools. It also makes sense that the attendings in Ginsburg and colleagues' study expected students to automatically know what to do; when one is embedded in a culture, the culture itself is very hard to see or articulate. Rather, one feels the culture, like an invisible hand that guides one's actions.

There is a recurring discussion in which I have been fortunate to be included at my institution and several others. It centers on what to do about a perceived crisis of professionalism among students. It typically ends up with some kind of strategy to identify and remediate the students who are either behaving unprofessionally, or are at risk for behaving unprofessionally. I once suggested that perhaps a parallel structure should be built to identify system issues that created opportunities for students to behave unprofessionally. The suggestion did not go over well. The fact remains that culture change is difficult, complex, unpredictable, and slow to evolve. It takes time to articulate what you can only at first feel. However, the system contains both powerful inhibitors and enablers of professionalism. Exciting work at the University of Indiana ${ }^{11}$ and elsewhere is attempting to understand and harness such enablers to create a lasting effect on the professional attitudes and behaviors of those who live and work in the system. Stark and colleagues' work in this issue of $J G I M^{7}$ demonstrates the promise that such system-based interventions can have for improving faculty feedback, a notoriously difficult process to change. My dream is that while the educational community struggles to learn how to assess and foster, on an individual level, such virtues as altruism and compassion, we will also engage in conversations aimed at defining how such virtues are realized at the level of a hospital, or a healthcare network, or the US healthcare system. For example, what would an "altruistic hospital" look and function like? What would it feel like to be a physician in such a hospital?

Clearly, there is much to be done when it comes to the topic of professionalism. The exciting work in this issue of JGIM points toward several directions in which the field is moving. My hope is that funding agencies, foundations, and the general public will have as much interest and stake in this topic as the scholarly community already does. Amid all of the technological advances occurring on an almost daily basis, the subject of professionalism is critically important, because it holds the potential to chart where healthcare has been, and where it is headed.

Corresponding Author: Paul Haidet, MD, MPH; 2002 Holcombe Blvd (152) Houston, TX 77030, USA (e-mail: phaidet@bcm.tmc.edu).

\section{REFERENCES}

1. ABIM Foundation, American Board of Internal Medicine, ACP-ASIM Foundation, American College of Physicians-American Society of Internal Medicine, European Federation of Internal Medicine. Medical professionalism in the new millennium: a physician charter. Ann Intern Med 2002;136:243-6.

2. Thompson LA, Dawson K, Ferdig R, Black EW, Boyer J, Coutts J, Black NP. The Intersection of Online Social Networking with Medical Professionalism. J Gen Intern Med 2008;23. DOI 10.1007/s11606-0080538-8.

3. Wear D, Zarconi J. Can compassion be taught? Let's ask our students. J Gen Intern Med 2008;23. DOI 10.1007/s11606-007-0501-0.

4. Hill-Sakurai LE, Lee CA, Schickedanz A, Maa J, Lai CJ. A professional development course for the clinical clerkships: developing a studentcentered curriculum. J Gen Intern Med 2008;23. DOI 10.1007/s11606008-0527-y

5. Fisher MA, Harrell HE, Haley H, Cifu AS, Alper E, Johnson KM, Hatem D. Between two worlds: a multi-institutional qualitative analysis of students' reflections on joining the medical profession. J Gen Intern Med 2008;23. DOI 10.1007/s11606-008-0508-1.

6. Ginsburg S, Lingard L, Regehr G, Underwood K. Know when to rock the boat: how faculty rationalize students' behaviors. J Gen Intern Med 2008;23. DOI 10.1007/s11606-007-0401-3.

7. Stark R, Korenstein D, Karani R. Impact of a 360-degree professionalism assessment on faculty comfort and skills in feedback delivery. J Gen Intern Med 2008;23. DOI 10.1007/s11606-008-0586-0.

8. Haidet P. Jazz and the 'art' of medicine: improvisation in the medical encounter. Ann Fam Med 2007;5:164-169.

9. Legassie J, Zibrowski EM, Goldszmidt MA. Measuring resident wellbeing: impostorism and burnout syndrome in residency. J Gen Intern Med 2008;23. DOI 10.1007/s11606-008-0536-x

10. Inui TS. A Flag in the Wind: Educating for Professionalism in Medicine. Washington, DC: Association of American Medical Colleges; 2003.

11. Cottingham AH, Suchman AL, Litzelman DK, Frankel RM, Mossbarger DL, Williamson PR, Baldwin DC Jr, Inui TS. Enhancing the Informal Curriculum of a Medical School: A Case Study in Organizational Culture Change. J Gen Intern Med 2008; Mar 14: Epub ahead of print. 